\title{
MODELAMIENTO DE FUENTE SÍSMICA DEL SISMO DEL QUINDÍO (COLOMBIA) DEL 25 DE ENERO DE 1999 USANDO INVERSIÓN DE FORMA DE ONDA DE CUERPO TELESÍSMICA
}

\author{
SEISMIC MODELING OF EARTHQUAKE SOURCE Quindío (COLOMBIA) FROM 25 JANUARY \\ 1999 USING WAVEFORM INVERSION teleseismic BODY
}

Lynette Vanessa Chavarría ${ }^{1}$, Helber García Camacho ${ }^{1}$ y Hugo Monsalve J. ${ }^{2}$

\footnotetext{
${ }^{1}$ Grupo Quimbaya, Universidad del Quindío, e-mail: vane_6358@hotmail.com; helbercam50@hotmail.com

${ }^{2}$ CEIFI, Grupo Quimbaya - Universidad del Quindío, e-mail: hugom@uniquindio.edu.co
}

\section{RESUMEN}

El sismo de Armenia - Quindío, Colombia $\left(\mathrm{M}_{\mathrm{w}}=6.2\right)$ del 25 de enero de 1999, es el sismo superficial más grande registrado instrumentalmente en el Eje Cafetero Colombiano. Se localizó en las inmediaciones del municipio de Córdoba (Quindío), a 17 kilómetros de la ciudad de Armenia. Se obtuvo mediante inversión de forma de onda el área y proceso de ruptura, con registros de desplazamiento de banda ancha con ondas en el rango de 5 a $0.01 \mathrm{~Hz}$. En dicho proceso de inversión se obtuvo un área de ruptura de $125 \mathrm{~km}^{2}$, una magnitud $\mathrm{M}_{\mathrm{w}}=6.0$ y una falla normal de desplazamiento lateral izquierdo de tipo unidireccional debido a que la presencia de réplicas indican que la ruptura de la falla creció con el tiempo hacia el noreste, sugiriendo una falla de este tipo; el mecanismo focal total predominante es de rumbo con componente normal, con buzamiento $\delta=48.9^{\circ}$, azimut $\phi=111.5^{\circ}$ y ángulo de deslizamiento $\lambda=-166.7^{\circ}$ producto de dos subeventos distribuidos a lo largo del área de ruptura, el primero de ellos se presenta en $\tau=8 \mathrm{~s} \mathrm{y} l=0 \mathrm{~km}$ y el segundo localizado en $\tau=26 \mathrm{~s} \mathrm{y} l=5 \mathrm{~km}$. Se obtuvo una profundidad del hipocentro $\mathrm{de} \mathrm{h}=20 \mathrm{~km}$, un momento sísmico de $1.26 \mathrm{x}$ $10^{25}$ dina*cm y una dislocación $\Delta \mathrm{u}=0.324 \mathrm{~m}$.

Se realizó la validación de los registros acelerográficos de la Red Sismológica Nacional de Colombia (RSNC) en dos estaciones, la primera de campo lejano (a una distancia de $141 \mathrm{~km}$ del sismo) y la segunda de campo cercano (a una distancia de $42 \mathrm{~km}$ del sismo) con registros sintéticos de movimiento del suelo, obteniendo un ajuste aceptable.

Palabras Clave: Sismo de Armenia - Quindío, Inversión de ondas de cuerpo telesísmica, mecanismo focal, validación. 


\begin{abstract}
In January 25th, 1999 Armenia earthquake $\left(\mathrm{M}_{\mathrm{w}}=6.2\right)$, up to now the largest shallow earthquake recorded instrumentally in the Colombian Eje Cafetero. It was located near Cordoba (Quindío) town, 17 kilometers from the Armenia (Colombia). The area and rupture process was obtained by inversion of teleseismic body waves, with broad band displacement records with wave ranges from 5 to $0.01 \mathrm{~Hz}$. This process allowed to obtain a rupture area of $125 \mathrm{~km}^{2}$, a magnitude $\mathrm{M}_{\mathrm{w}}=6.0$ and a left lateral strike - slip fault of unidirectional type. Since the presence of aftershocks indicate a that the fault rupture grew over time to the northeast, suggesting a fault of this type. The prevalent focal mechanism is strike - slip with a dip $\delta=48.9^{\circ}$, an azimuth $\phi$ $=111.5^{\circ}$ and a slip angle $\lambda=-166.7^{\circ}$ as a result of two subevents distributed along the rupture area; the first one occurred in $\tau=8 \mathrm{~s}$ y $l=0 \mathrm{~km}$ and the second one located at $\tau=26 \mathrm{~s} \mathrm{y} l=5 \mathrm{~km}$. A hypocenter depth about 20 $\mathrm{km}$ was obtained, a seismic moment of $1.26 \times 10^{25}$ dina* $\mathrm{cm}$ and a dislocation $0.324 \mathrm{~m}$.

The validation was made with acelerographics records of Red Sismológica Nacional de Colombia (RSNC) in two stations, the first one a far- field (at $141 \mathrm{~km}$ of distance to the earthquake) and the second one a nearfield station (at $42 \mathrm{~km}$ of distance to the earthquake). With synthetic records of ground motion, was obtained an acceptable fit between the model and the recorded data.
\end{abstract}

Keywords: Armenia - Quindío (Colombia) earthquake, inversion of teleseismic body waves, focal mechanism, validation.

\section{INTRODUCCIÓN}

El sismo del Quindío (Colombia) del 25 de Enero de 1999 de magnitud $\mathrm{M}_{\mathrm{w}}=6.2$, ocasionado por una falla superficial en la corteza continental $(1,2)$ es el sismo más grande registrado instrumentalmente en el eje cafetero colombiano, se localizó en inmediaciones del municipio de Córdoba (Quindío), a 17 kilómetros de la ciudad de Armenia. La fuente sísmica que tiene que ver con el origen de este sismo, es una fuente que aporta significativa-mente a la sismicidad y a la amenaza sísmica de la región. La evolución tectónica de la estructura cortical en el departamento del Quindío, ha estado sometida a un estado de esfuerzos principalmente compresionales, producto de la convergencia de la placa Nazca subducida bajo la placa Suramericana (3). Este sismo fue abordado con inversión de ondas de cuerpo propuesto por Nabelek (4), pero no fueron analizados los procesos de ruptura de la fuente sísmica.

El formalismo de comparar sismos sintéticos y sismos observados es conocido como modelamiento de formas de onda, la modelación es un proceso iterativo en el cual los residuales de las amplitudes del sismograma sintético y el sismograma observado se minimizan ajustando la fuente o plano de falla. Para sismos con $M_{w}>6.0$, es necesario explicar el proceso de ruptura, debido a que en campo cercano el efecto de la fuente sísmica hace más complejo la propagación de las ondas de cuerpo.

Se han desarrollado diferentes métodos para invertir on- das de cuerpo, por ejemplo, en el método de inversión propuesto por Nabelek (4), el mecanismo de subeventos es determinado por un método iterativo de mínimos cuadrados. Barker y Langston (5) desarrollaron la técnica de inversión generalizada utilizando el momento tensor. Koyama (6) invirtió ondas de cuerpo telesísmicas de período largo a una secuencia de tiempo del momento tensor. Hirata y Kawasaki (7) analizaron ondas de cuerpo para sismos profundos e investigaron el cambio en el mecanismo de falla durante el proceso de fuente.

La complejidad de la función temporal de la fuente refleja la heterogeneidad en las propiedades de la roca en la zona de falla, que se caracteriza por la presencia de asperezas y barreras, este conocimiento permite comprender el plano de ruptura y el proceso bidireccional o unidireccional que sigue la ruptura de la fuente. El proceso de ruptura explica la parada súbita del terremoto. Es necesario conocer seis parámetros, el momento sísmico, el azimut, el buzamiento, el ángulo de deslizamiento, el tiempo de levante de la fuente y el tiempo de ruptura.

Kikuchi and Kanamori $(8,9)$ propusieron que el patrón de ruptura es presentado por una secuencia de subeventos distribuidos en el plano de falla. En el método, se invierten ondas de cuerpo para obtener el mecanismo y patrón de ruptura de eventos complejos, los mecanismos de los subeventos se permiten que varíen durante la secuencia, aunque esto puede inestabilizar la inversión, pero reproduce fielmente el proceso de ruptura 
de la fuente sísmica. En los métodos anteriores a 1991 propuestos por Kikuchi and Kanamori se usaron ondas $\mathrm{P}$, en el nuevo método se usan ondas $\mathrm{P}, \mathrm{SH}, \mathrm{SV}$ y PP invertidas simultáneamente, se utiliza un modelo de estructura de multicapas, es decir estratificado, además se utiliza la matriz de propagación de Haskell como se describe en Bouchon (10) y Haskell $(11,12)$.

Con este método se han invertido sismos complejos como: el sismo del 4 de febrero de 1976 en Guatemala $\left(\mathrm{M}_{\mathrm{w}}=7.5\right)$, el cual es uno de los sismos superficiales más devastadores ocurrido sobre una falla transformante, con un promedio de dislocación de un metro observado a la largo de $200 \mathrm{~km}$ de longitud en la sección curva de la falla Motagua (13). El sismo del 7 de diciembre de 1988 en Spitak, Armenia $\left(M_{w}=6.8\right)$, que a pesar de su tamaño moderado causó el mayor desastre sísmico desde el sismo de 1976 en Tanshan, China. Desde la complejidad de la fuente puede haber un importante comportamiento en el poder destructivo de un sismo sobre el patrón de deformación cosísmico en un ambiente tectónico complejo.

El presente trabajo muestra los resultados de la inversión de ondas de cuerpo desarrollada por Kikuchi and Kanamori $(8,9)$, el proceso de ruptura, tipo de falla, momento sísmico y las características de la fuente del sismo Armenia-Quindío, Colombia del 25 de enero de 1999; además, de la validación de los registros de aceleraciones de la Red Sismológica Nacional de Colombia utilizando el algoritmo de Spudich y Xu (14).

\section{METODOLOGÍA Y PROCESAMIENTO DE DA- TOS}

\section{Inversión de ondas de cuerpo telesísmicas}

La metodología empleada se basó en la inversión de forma de onda de Kikuchi and Kanamori $(8,9)$, que consistió en encontrar el proceso de ruptura de la fuente sísmica obtenida por una secuencia de subeventos con mecanismos variables. Una vez caracterizados los sismogramas observados en distancias epicentrales $30^{\circ}<\Delta<95^{\circ}$, se ajustaron iterativamente con los sismogramas sintéticos hasta determinar los parámetros de la fuente y su ruptura.

Para el funcionamiento del algoritmo de inversión de ondas de cuerpo de Kikuchi y Kanamori, se utilizó para el pre-procesamiento de señales el software SAC (15), para descartar las estaciones de la red IRIS que no con- taban con un registro aceptable de la señal sísmica y prepararla para abordar el proceso de inversión de onda de cuerpo de forma correcta y obtener la ruptura de la fuente sísmica para el sismo propuesto. La implementación de estos algoritmos exige la calibración de la sensibilidad de los parámetros y requieren los análisis espectrales previos para las suposiciones del área de ruptura inicial y el tamaño de la malla para que sean adecuadas con la longitud de ondas de cuerpo. Igualmente el filtrado de señales adecuados de acuerdo a la relación ruido/ señal, producto de un intenso trabajo de inspección visual con los datos de las señales sísmicas, permitieron un ajuste adecuado.

\section{Algoritmo para el cálculo de sismogramas sintéticos de movimiento del suelo}

Para el cálculo de las velocidades se utilizó el algoritmo de Spudich y Xu (14) y un algoritmo diseñado en lenguaje Matlab (dshaCompsyn) para calcular y graficar las velocidades generadas por la fuente sísmica basados en los parámetros de falla, área, tipo de ruptura, entre otros. En la simulación se establecen las características del medio en el que se va a simular la señal sísmica, para esto se utilizan las aplicaciones OLSON, XOSLON, TFAULT, SLIP y SEESLO, cada aplicación utiliza la información de la aplicación que precede como se observa en la figura 1. OLSON, calcula las funciones de Green para un modelo de Velocidad-Densidad elegido por el usuario; XOLSON, reestructura la salida (binaria) de los archivos generados por Olson; TFAULT, calcula las tracciones en el plano de falla para un conjunto de puntos receptores en donde se quiere calcular los sismogramas; SLIP, convoluciona un modelo de ruptura dado con las tracciones en la falla calculada anteriormente (en el archivo de Tfault); SEESLO, transforma mediante la transformada de Fourier las formas de onda (domino de la frecuencia), escribe los archivos ascci para las series de tiempo y se generan los gráficos de las formas de onda en archivos post-script (se pueden filtrar las señales).

El algoritmo dshaCompsyn es un programa que calcula las velocidades en un punto específico definido por el usuario (basándose en el paquete de subrutinas Compsyn) y genera todos los archivos de entrada para las subrutinas de Compsyn, además, clasifica toda la información generada por la fuente sísmica en carpetas separadas con el nombre de la misma (16). 


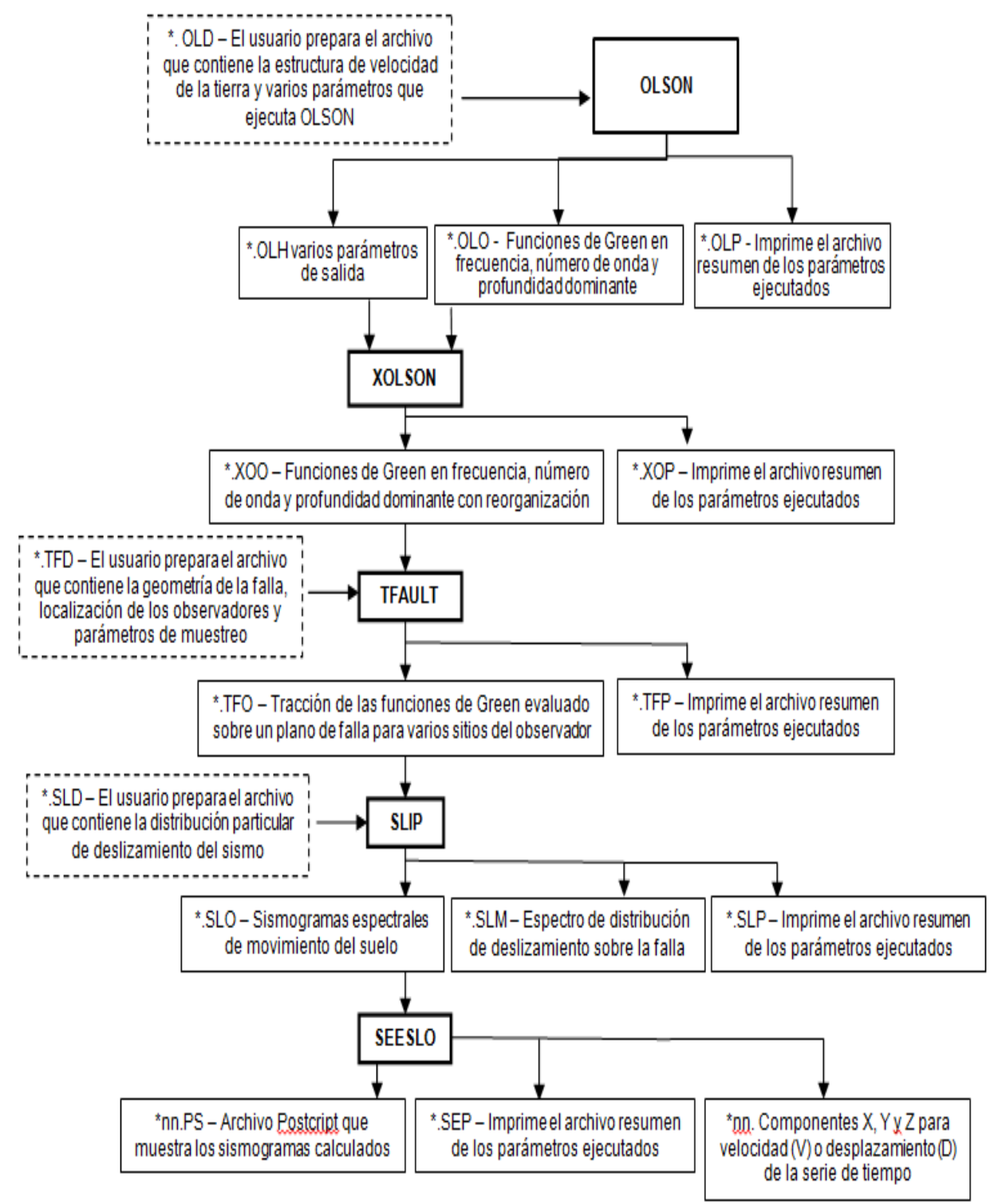

Figura 1. Diagrama de flujo que muestra la secuencia en la cual son ejecutadas las rutinas y muestra los archivos usados y/o creados por la aplicación. Los recuadros con línea punteada son archivos preparados por el usuario. Los recuadros con línea gruesa son las aplicaciones. Los recuadros con línea delgada son los archivos creados por la aplicación (Tomado de Manual Compsyn. Spudich, P. and Xu, L., 2002.)

\section{Procesamiento de datos}

Se usaron ondas de cuerpo telesísmicas $\mathrm{P}$ y SH de dieciséis [16] estaciones de Incorporated Research Institutions for Seismology (IRIS). La red IRIS tiene una banda ancha con velocidad de respuesta plana en un amplio rango de frecuencia. Las estaciones utilizadas tienen una distancia epicentral entre 30 y $95^{\circ}$ y la cobertura en el hemisferio norte es buena, hacia el sur es pobre puesto que sólo se cuenta con una estación y hacia el sureste se cuenta con buena cobertura como se observa en la figura 2. El procedimiento para preparar los datos previos a la inversión fue : [1] Se eliminó la media mediante la corrección por línea base, [2] haciendo uso de las tablas de Jeffreys - Bullen (17) se calculó el tiempo de viaje teórico de la onda $\mathrm{P}$ acompañado de una inspeccion visual de la señal y se marcaron los arribos de la onda P; se descartaron las señales en las que no había coincidencia entre el tiempo teórico y el observado, [3] los registros fueron digitalizados e interpolados en un intervalo de una muestra por segundo (1 mps), [4] se les aplicó un filtro Butterworth para altas frecuencias 
de corte de 0.01 y $0.015 \mathrm{~Hz}$ a eventos que presentaban ruidos a bajas frecuencias, [5] se rotaron los eventos de acuerdo a la convención de Aki y Richards (18).

El procesamiento de las señales fue realizado utilizando el software SAC (15), un programa interactivo diseñado para el estudio de señales secuenciales, se descartaron las estaciones que presentaban señales muy ruidosas y aquellas en que su distancia epicentral no estaban entre $30^{\circ}$ y $95^{\circ}$. En la tabla 1 se enlistan los parámetros de las estaciones y las fases $\mathrm{P}$ y SH utilizadas para el análisis.

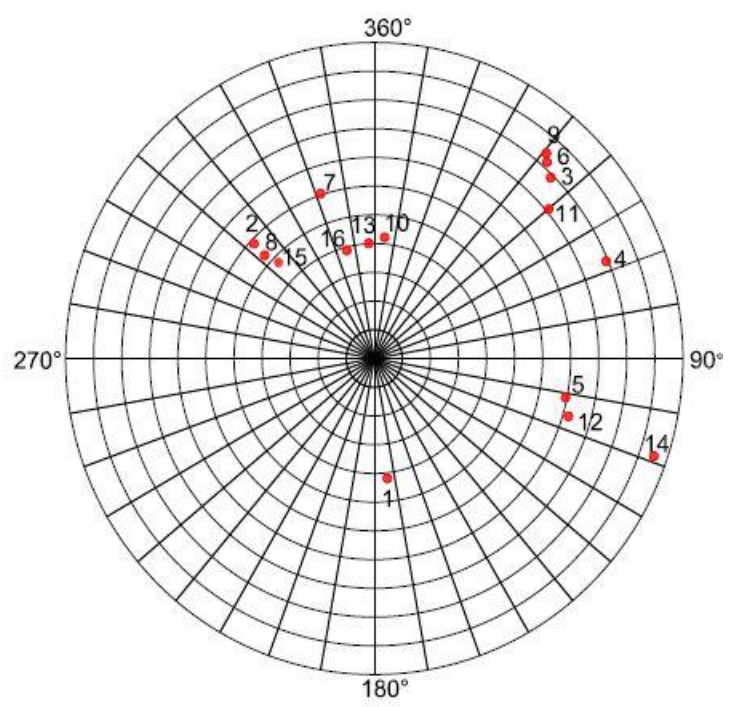

Figura 2. Estaciones Sísmicas usadas en el análisis

Tabla 1. Parámetros de las estaciones

\begin{tabular}{cccccccl}
\hline \multicolumn{1}{c}{ ESTACION } & RED & AZIMUT & BACKAZIMUT & DISTANCIA & FASE & PESO \\
\hline 1 & PEI & IRIS & 173.6 & 351.75 & 4196.2 & P, SH & $1.0,0.1$ \\
2 & SCZ & IRIS & 313.6 & 116.22 & 5859.9 & P, SH & $1.0,0.05$ \\
3 & SSB & IRIS & 44.96 & 266.23 & 8897.3 & P, SH & $1.0,0.1$ \\
4 & TAM & IRIS & 67.74 & 270.75 & 8928.4 & P, SH & $1.0,0.1$ \\
5 & ASCN & IRIS & 101.27 & 279.22 & 6950.4 & P, SH & $1.0,0.1$ \\
6 & BFO & IRIS & 41.996 & 268.54 & 9201.3 & P, SH & $1.0,0.05$ \\
7 & FFC & IRIS & 341.61 & 147.17 & 6054.02 & P, SH & $1.0,0.1$ \\
8 & PFO & IRIS & 312.58 & 118.4 & 5307.1 & P, SH & $1.0,0.05$ \\
9 & GRFO & IRIS & 40.64 & 270.56 & 9413.2 & P, SH & $1.0,0.1$ \\
10 & HRV & IRIS & 4.998 & 186.75 & 4234.7 & P, SH & $1.0,0.1$ \\
11 & PAB & IRIS & 50.07 & 261.44 & 8101.9 & P, SH & $0.5,0.1$ \\
12 & SFJ & IRIS & 106.21 & 207.88 & 7203.9 & P, SH & $1.0,0.1$ \\
13 & SSPA & IRIS & 357.2 & 176.33 & 4012.7 & P, SH & $1.0,0.1$ \\
14 & TSUM & IRIS & 108.83 & 273.12 & 10525.7 & P, SH & $1.0,0.01$ \\
15 & TUC & IRIS & 314.42 & 122.77 & 4777.3 & P, SH & $1.0,0.05$ \\
16 & WCI & IRIS & 345.39 & 161.39 & 3890.39 & P, SH & $1.0,0.05$ \\
\hline
\end{tabular}

\section{Inversión de ondas de cuerpo telesísmicas}

El método iterativo de deconvolución desarrollado por Kikuchi and Kanamori $(8,9)$, permite cambios en los mecanismos durante la secuencia de ruptura. El proceso de fuente es representado como una secuencia de subeventos, cada uno especificado por un momento tensor, un tiempo de inicio y localización.

Para emplear dicho método se utilizó una ventana de 90 segundos después del arribo de la onda P para la inversión de onda.

\section{Esquema de la malla y Funciones de Green}

Para la localización de los subeventos se tomaron 6 puntos distribuidos en la dirección $\mathrm{N} 4^{\circ} \mathrm{W}-\mathrm{S} 4^{\circ} \mathrm{E}$, a una separación de $2.5 \mathrm{~km}$ y 5 puntos a lo largo del buzamiento a la misma separación (ver figura 3 ) basados en estudios anteriores.

El primer paso es el cálculo de las funciones de Green para el momento tensor elemental en una estructura de capas. En la tabla 2 se muestra la estructura de la fuente y los sitios receptores usados para el cálculo de las funciones de Green. La estructura de la fuente y receptor cercanos corresponden a la ciudad de Armenia. Las funciones fuente y receptor fueron calculadas usando la matriz de propagación de Haskell de la misma forma descrita por Bouchon (10) y Haskell $(11,12)$.

\section{Peso de las estaciones}

Se aplicaron factores de peso a las formas de onda sintéticas y observadas de 1 y 0.1 para la onda $\mathrm{P}$ y $\mathrm{SH}$ respectivamente de acuerdo a las recomendaciones de Kikuchi and Kanamori $(8,9)$, y fueron ajustados considerando la cobertura azimutal y la calidad de los registros observados. El factor de peso de la Onda P y SH registrada por la estación PEL fue equivalente al usado en otras estaciones ubicadas es zonas con mejor cobertura azimutal debido a que la pobre calidad de los registros no compensaba la escasa cobertura en el sur.

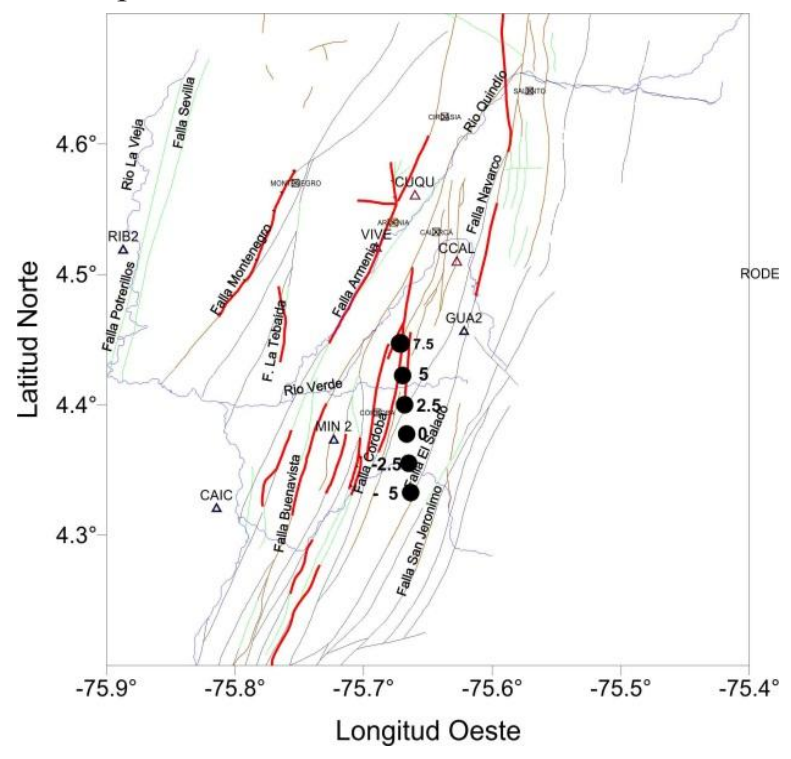

Figura 3. Esquema de la malla. Cinco puntos con espaciamiento $2.5 \mathrm{~km}$ sobre la línea del rumbo 
Tabla 2. Estructura de la fuente y receptor

\begin{tabular}{cccc}
\hline $\boldsymbol{\alpha}(\mathrm{km} / \mathrm{s})$ & $\beta(\mathrm{km} / \mathrm{s})$ & $\rho\left(\mathrm{g} / \mathrm{cm}^{3}\right)$ & Espesor $(\mathrm{km})$ \\
\hline \multicolumn{4}{c}{ Estructura de la fuente } \\
\hline $\mathbf{4}$ & 2.14 & 2.35 & 1 \\
$\mathbf{5 . 6}$ & 3.23 & 2.7 & 5 \\
$\mathbf{6 . 5}$ & 3.75 & 2.85 & 30 \\
$\mathbf{7 . 8}$ & 4.4 & 3.3 & 10 \\
$\mathbf{8 . 1}$ & 4.4 & 3.3 & 200 \\
\hline \multicolumn{4}{c}{ Estructura del receptor } \\
\hline $\mathbf{5 . 5 7}$ & 3.36 & 2.65 & 15 \\
$\mathbf{6 . 5}$ & 3.74 & 2.85 & 18 \\
$\mathbf{7 . 8}$ & 4.4 & 3.3 & 0 \\
\hline
\end{tabular}

\section{Equilibrio entre la sincronización ( $\tau$ ) y el mecanismo}

El parámetro $p$ representa el tiempo $(\tau)$, la localización $(l)$ a lo largo del rumbo y la profundidad h de los sub eventos. En este análisis se usó extensivamente el diagrama $\tau-l$ descrito por Kikuchi and Kanamori (9). Primero, un subevento fue colocado sobre la malla de puntos en el plano $\tau-l$; la correlación fue calculada en el proceso de inversión y el mecanismo resultante fue graficado en una malla de puntos sobre el diagrama $\tau$ l. En los cálculos se usó una función temporal triangular simétrica con un tiempo de levante (rise time) de $4.5 \mathrm{~s}$ y una duración total de $9 \mathrm{~s}$. Esta función temporal fue usada en todas las iteraciones.

En la figura 4 (a) se muestra el mapa de contorno de la función correlación $\Psi(\tau, l, \mathrm{~h}=20 \mathrm{~km})$ sobre el plano $\tau-1$ para el sistema básico de doble-par. La línea sólida tipo escalera que se observa en la figura 4 (a) representa la ruptura frontal con una velocidad de ruptura de 3 $\mathrm{km} / \mathrm{s}$ (se asumió que los subeventos se encuentran localizados a la derecha de la ruptura frontal sobre el plano $\tau$ - 1. figura 4 (c) muestra los mecanismos de los Subeventos y el mecanismo total. El mecanismo del primer subevento localizado en $\tau=8$ s y $l=0 \mathrm{~km}$; el segundo subevento localizado en $\tau=26$ s y $l=5 \mathrm{~km}$.

Una vez determinada la secuencia de subeventos, se fijaron sus mecanismos, localización y se liberaron las restricciones de todos los subeventos (teniendo la misma función temporal de fuente). La función temporal de fuente [figura 7 (a)] se representó con la superposición de funciones de fuente de triángulos angostos; se usó una función triangular con una base de ancho de nueve (9) s y luego se distribuyó en intervalos iguales a un (1) s alrededor del tiempo de inicio de cada subevento, determinado mediante el proceso de iteración (la altura de los triángulos fueron determinadas por medio del método lineal de mínimos cuadrados).

La figura 5 muestra el mejor ajuste de doble-cupla puesto en una malla de puntos separados $2.5 \mathrm{~km}$ en el espacio y $10 \mathrm{~s}$ en el tiempo.

La solución final corresponde al mejor ajuste $(\Delta=$ $0.2726)$ producto del refinamiento en el proceso de inversión en el que se reajusta la función temporal de cada uno de los subeventos tomando el intervalo de tiempo en el que observa una buena correlación [ver figura 4

\section{Quindio, Colombia 99/01/25}
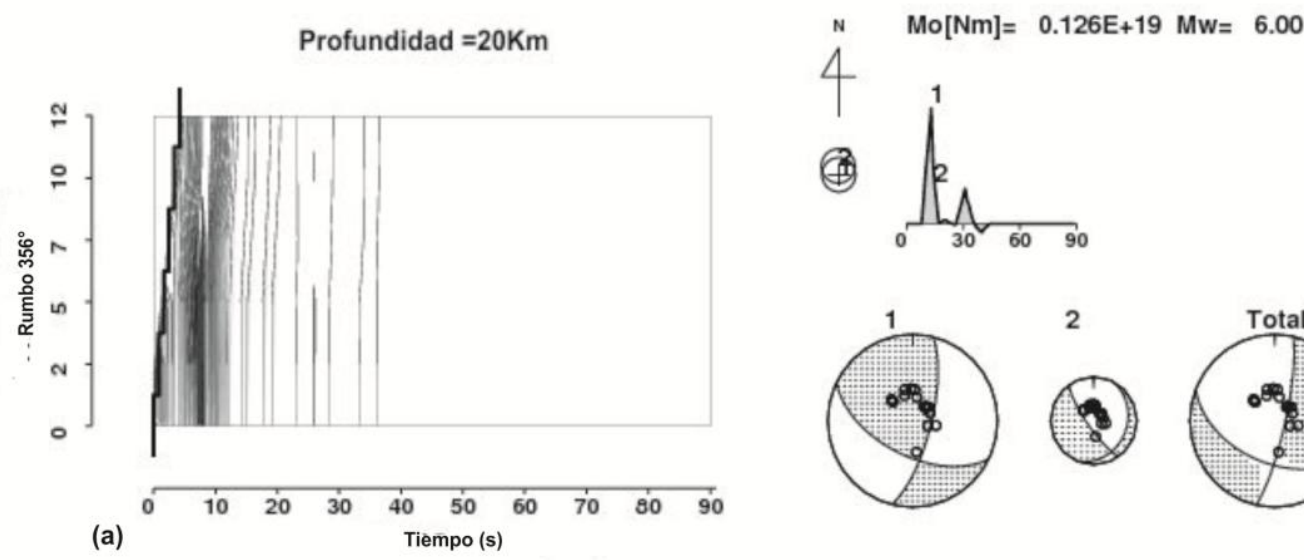

(b)
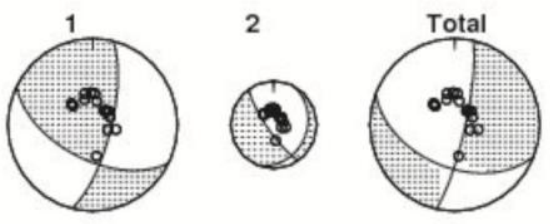

(C)

Figura 4. (a) Mapa de contorno de la función correlación del sistema básico doble-cupla; (b) Función temporal de fuente y (c) mecanismos de los subeventos y mecanismo total 


\section{Quindio, Colombia 99/01/25}

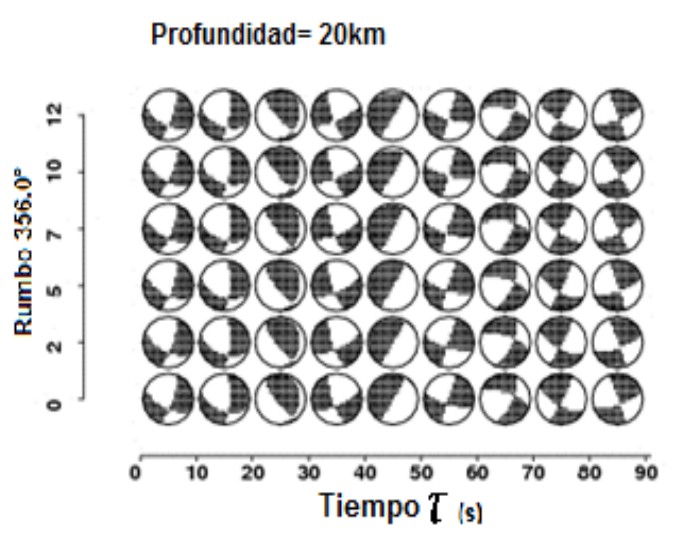

Figura 5. Mejor ajuste de doble-cupla en una malla de puntos cada $10 \mathrm{~s}$

(a)]. Para el primer subevento se observa una buena correlación en el intervalo $\tau=8-18 \mathrm{~s}$, el mecanismo encontrado es fijado y se prueban diferentes tiempos $\tau$ en el intervalo, cada uno con la misma función temporal triangular usada en la inversión de ondas de cuerpo [(tiempo de levante (rise time) de $4.5 \mathrm{~s}$ y tiempo de ruptura de $9 \mathrm{~s}$ )], este proceso se hace simultáneamente con el segundo subevento. Para éste, se toma el intervalo $\tau=26-35 \mathrm{~s}$. Además, este proceso permite reajustar el mecanismo y el tiempo para el cual ocurre un subevento reduciendo el error. Los parámetros finales de la fuente se muestran en la tabla 3.

En la Figura 6 se presentan las formas de onda sintéticas para el modelo final, las cuales son comparadas con las formas de onda observadas.

Los juegos de formas de onda son buenos para todas las estaciones excepto las SH de: SCZ, PFO y TSUM.

La distribución de los subeventos encontrados en el proceso de inversión muestra que la ruptura se propaga hacia el norte como se observa en la figura 7 (b) las zonas más oscuras indican la ocurrencia de los sube-

Tabla 3. Parámetros de fuente para la solución final

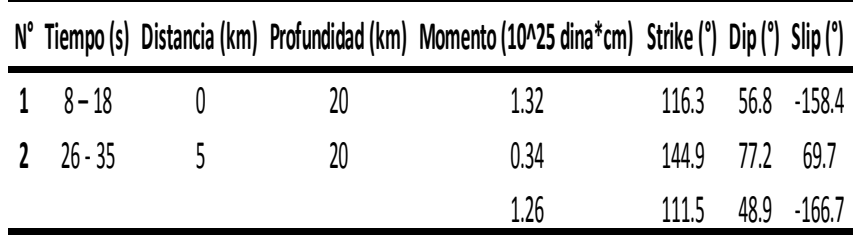

ventos $(\mathrm{l}=0 \mathrm{y} \mathrm{l}=5 \mathrm{~km})$ distribuidos a lo largo del rumbo de izquierda a derecha señalando una ruptura de tipo unidireccional debido a que la presencia de réplicas indican que la ruptura de la falla creció con el tiempo hacia el noreste, sugiriendo una falla de este tipo, siendo consistente con la encontrada por Monsalve y Vargas2. El tensor suma de todos los subeventos predominante es de rumbo con componente normal. El momento escalar es 1.26x1025 dina*cm. Se obtuvieron dos subeventos, el primero de deslizamiento hacia el rumbo que ocurre durante los primeros $18 \mathrm{~s}$ (subevento 1) con una duración efectiva de la ruptura de $10 \mathrm{~s}$; el segundo de buzamiento hacia el rumbo iniciando alrededor de los $26 \mathrm{~s}$ después del primer subevento, con una duración efectiva de la ruptura de $9 \mathrm{~s}$ [ver figura 7 (c)]. Las zonas más claras indican sitios donde la roca no rompió durante el proceso de ruptura "barreras". Das y Aki19 suponen un estado de esfuerzos homogéneos en la falla en la que existen barreras originadas por cambios en la litología, plegamientos bruscos, etc. que interfieren con el frente de ruptura, éstas pueden romper o permanecer irrompibles después del sismo. Estas zonas pueden romper luego debido al cambio de esfuerzo provocando réplicas.

\section{Distribución de réplicas sobre el plano de falla}

Para verificar que las réplicas distribuidas espacio temporalmente se localizan sobre el plano de ruptura obtenido durante el proceso de inversión de ondas de cuerpo de Kikuchi and Kanamori [mapa de contorno figura 7 (b)], éste se proyectó horizontalmente y se graficó junto a las réplicas del sismo del Quindío del 25 de enero de 1999 obtenidas del catálogo de eventos sísmicos del observatorio sismológico de la universidad del Quindío localizados durante el periodo de febrero a noviembre de 1999 ver figura 8 y 9.

La distribución de réplicas del mes de febrero llena el plano de falla y se puede observar el viaje de sur a norte; en los meses marzo, mayo, junio y julio se observa una leve disminución de réplicas dentro del plano de falla; en el mes de agosto se observa disminución en la sismicidad, sin embargo, aún hay una considerable cantidad de réplicas dentro del plano de falla; y en septiembre y octubre-noviembre se presenta una considerable disminución de la sismicidad acompañada de una mayor dispersión espacial de las réplicas posiblemente producto del cambio de esfuerzos en la zona. 


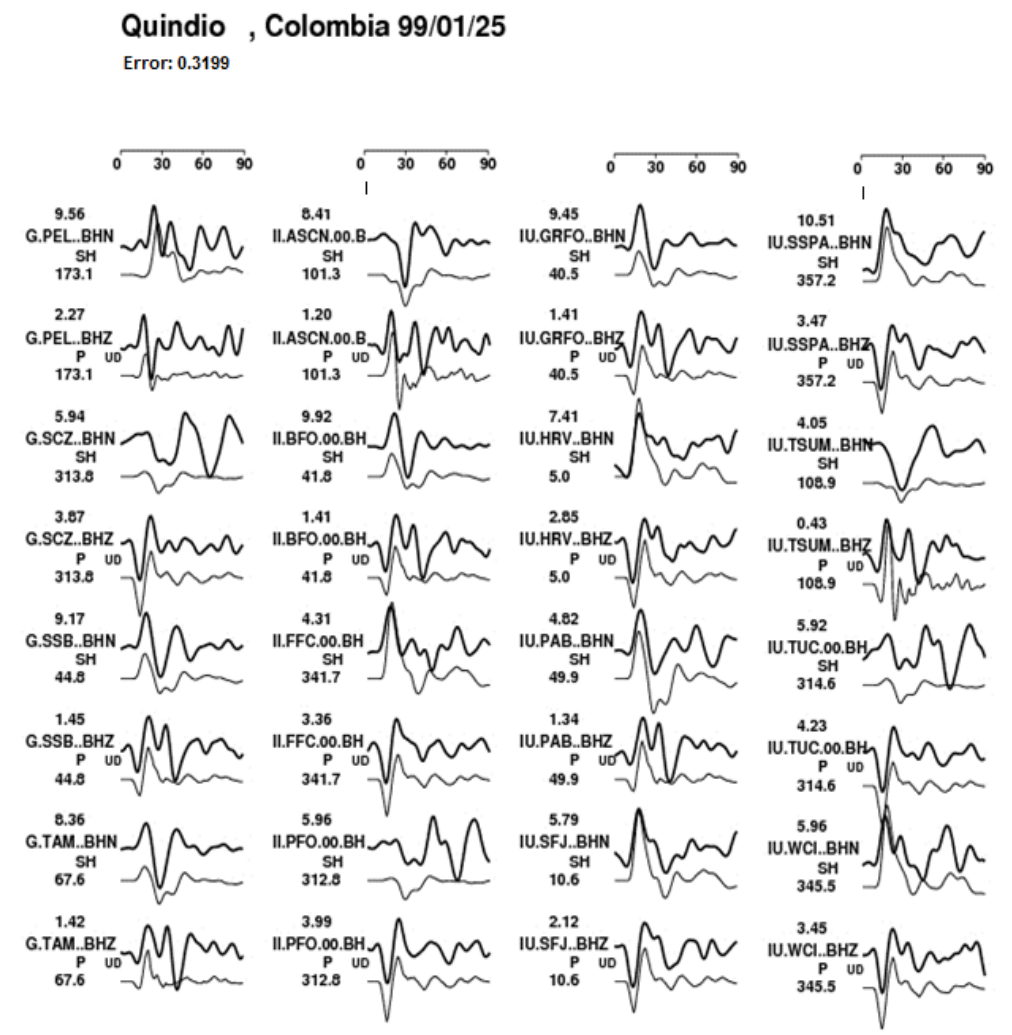

Figura 6. Comparación del registros observados (traza superior) y sintéticos (traza inferior) para la solución final. El valor superior de cada componente corresponde a la amplitud de la señal y el azimut el valor inferior.

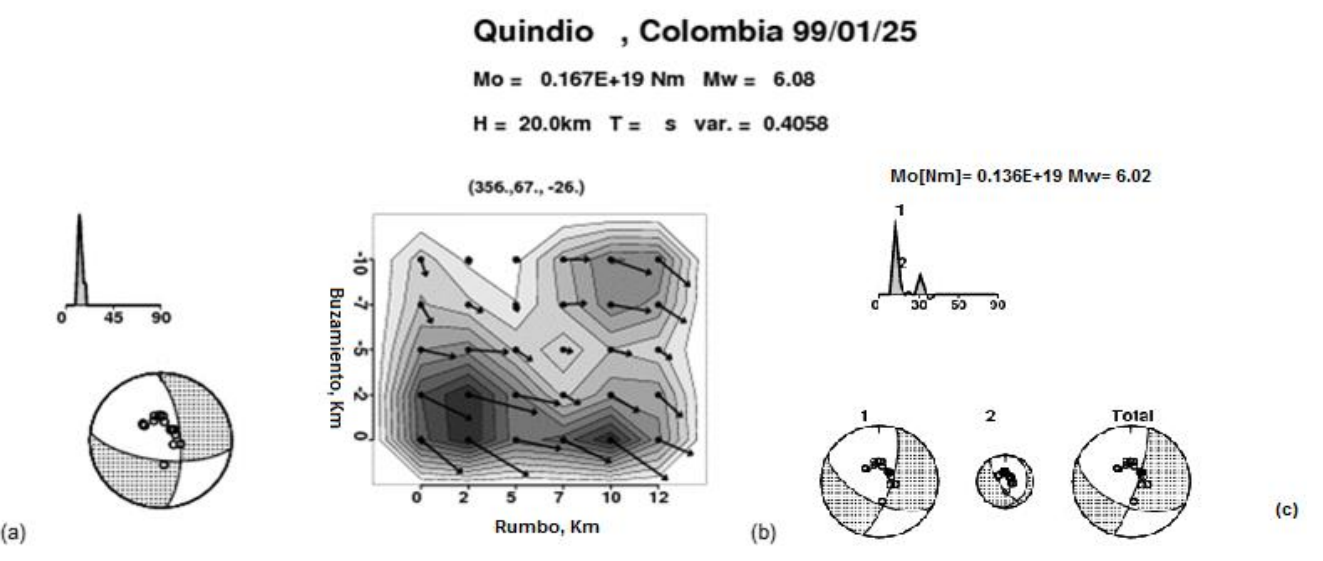

Figura 7. (a) Función temporal y mecanismo total; (b) Mapa de contorno de la distribución de Slip sobre el plano (strike - dip); (c) Arriba: función temporal. Abajo: Mecanismos de los subeventos y mecanismo total 


\section{Cálculo de sismogramas sintéticos de movimiento} del suelo

La simulación de las señales sísmicas es una de las formas de validar los registros de velocidad de un sismo en diferentes estaciones. En la simulación se tienen en cuentan características de la fuente (el momento sísmico, el azimut, el buzamiento, el ángulo de deslizamiento, el tiempo de levante de la fuente y el tiempo de ruptura) y un modelo de velocidad $1 \mathrm{D}$ acorde a la zona de estudio. Por lo tanto, la validez de la señal simulada
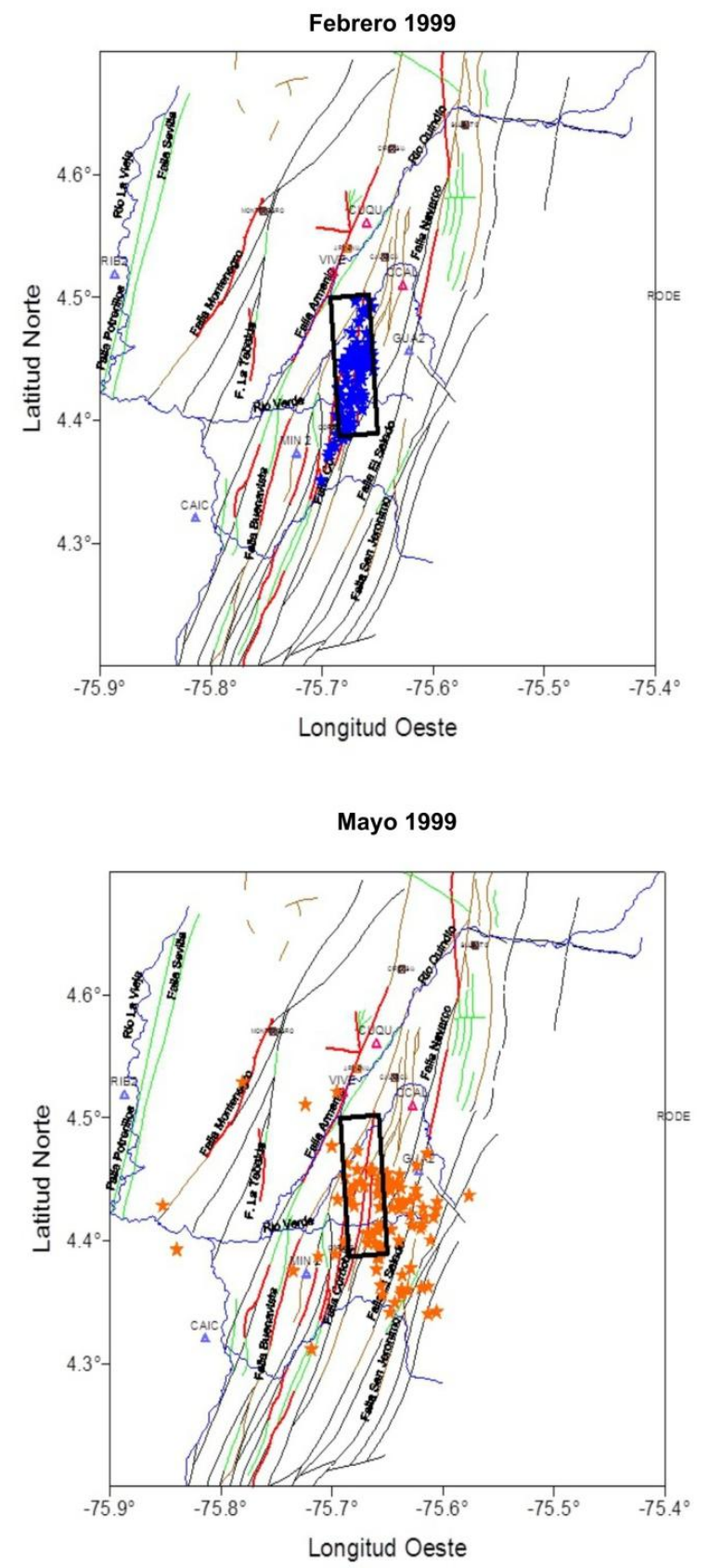

radica en que las amplitudes de los registros presenten similitud con dicha señal, además, que mediante observación se determine la coherencia en la forma de onda de ambas señales teniendo presente las limitaciones del modelo predictivo.

\section{Validaciones}

Con el fin de validar los registros acelerográficos del sismo del Quindío del 25 de enero de 1999, $\mathrm{Mw}=6.0$, obtenida en la inversión de ondas de cuerpo de Kikuchi
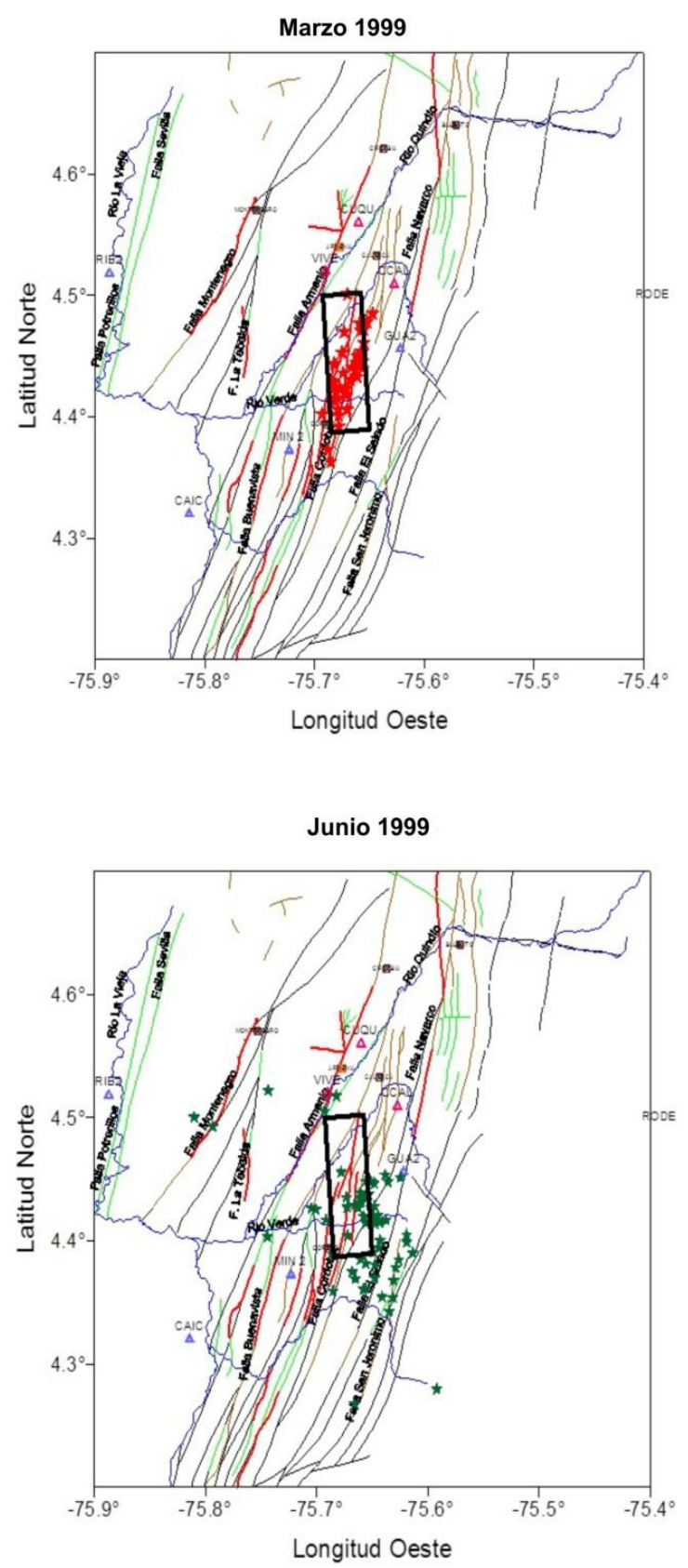

Figura 8. Distribución de réplicas sobre el plano de falla correspondiente a los meses febrero, marzo, mayo y junio de 1999 (el recuadro negro representa el plano de falla proyectado) 

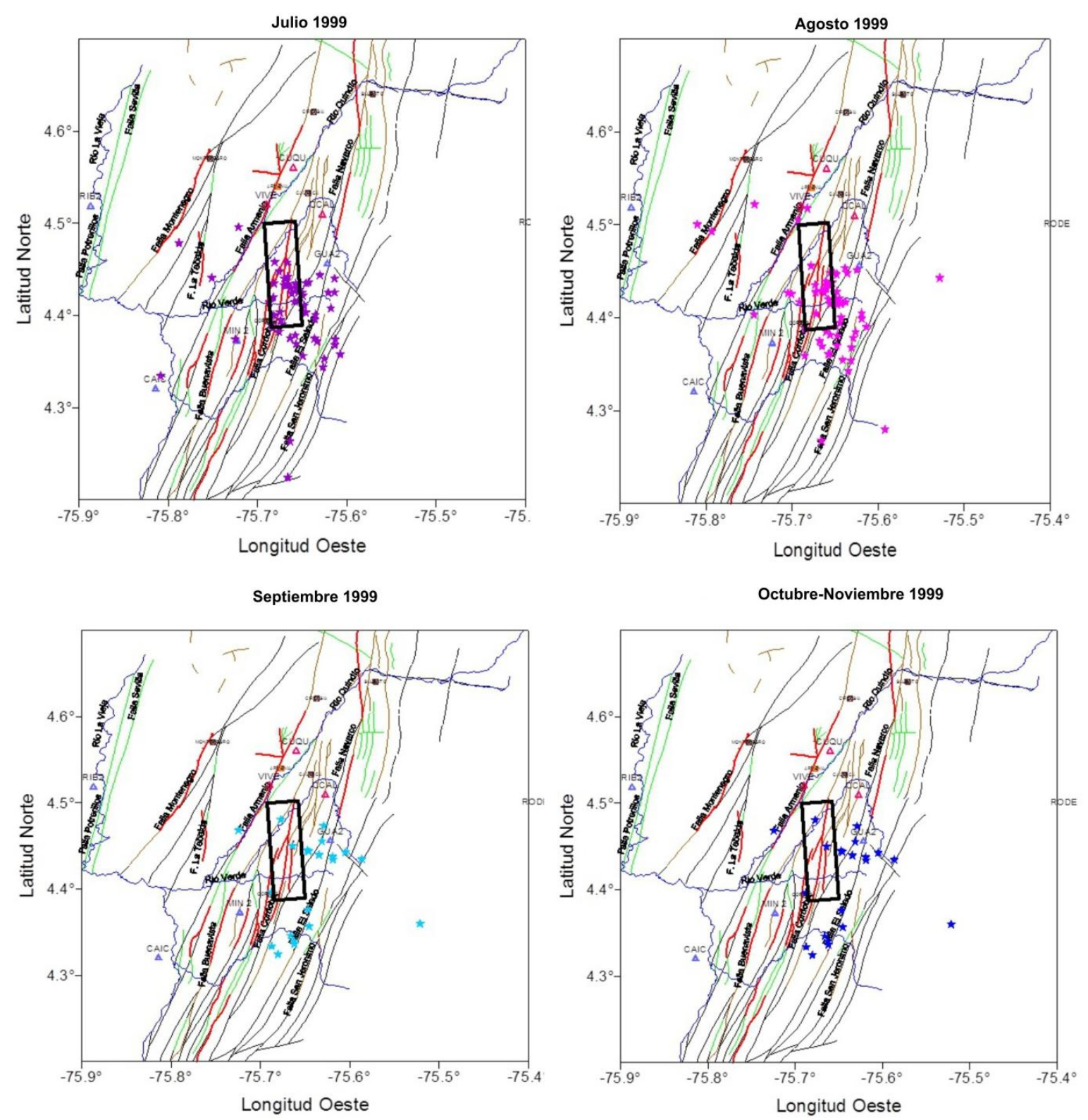

Figura 9. Distribución de réplicas sobre el plano de falla correspondiente a los meses julio, agosto, Septiembre y Octubre - Noviembre de 1999 (el recuadro negro representa el plano de falla proyectado)

and Kanamori. Se tomaron dos estaciones, teniendo en cuenta las distancias de registro del sismo, es decir, una estación cercana y una lejana del mismo, lo cual permite tener una percepción más adecuada de la validez de la simulación al tener diferentes casos en la comparación. Las estaciones seleccionadas para realizar estas comparaciones fueron las acelerográficas de la Red Sismológica Nacional de Colombia (RSNC) ANDES Y PERERO, las cuales se encuentran a una distancia del sismo de $141 \mathrm{~km}$ y $42 \mathrm{~km}$ respectivamente. De acuerdo a los resultados obtenidos con la modelación del algoritmo de Spudich y Xu (14), las validaciones se realizaron con registros en velocidad, por lo tanto, se procesó la señal registrada en aceleración para obtener registros en velocidad.

La simulación de las señales se realizó en los sitios donde se encuentran ubicadas las estaciones ANDES y PERERO bajo una frecuencia de trabajo de $5 \mathrm{~Hz}$, la geometría del plano de falla e información de la fuente obtenida con la inversión de ondas de cuerpo de Kikuchi and Kanamori. Para los registros simulados y observados se utilizó un filtro de 1 a $5 \mathrm{~Hz}$. Las tres componentes de la señal registrada por las estaciones ANDES Y PERERO son comparadas (en amplitud) con las tres componentes de las señales simuladas respectivamente 
(ver figura 10) y se encontraron diferencias entre las amplitudes del observado y el simulado, posiblemente producto de efectos de sitio que no son tenidos en cuenta en el algoritmo Spudich y Xu (14). en la zona de los observadores (ANDES y PERERO) debido a la amplificación del registro.
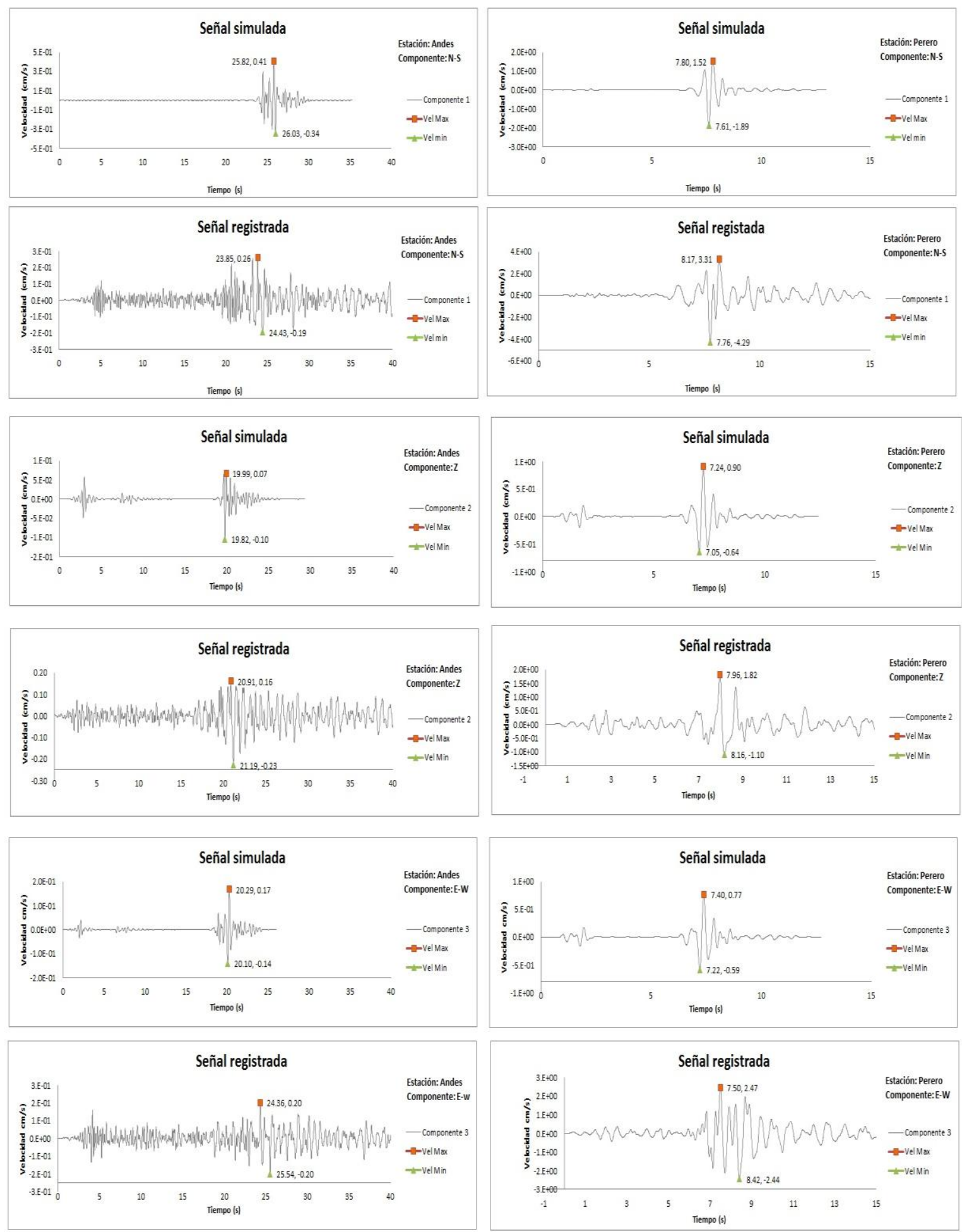

Figura 10. Comparación señal simulada con las tres (3) componentes (N-S, Z y E-W) de la señal registrada del sismo de Armenia-Quindío, Colombia del 25 de enero de 1999 (Mw=6.0). por las estaciones: (a) Estación Andes ubicada a 141 km de la localización del sismo, (b) Estación Perero ubicada a $42 \mathrm{~km}$ de la localización del sismo 


\section{RESULTADOS Y DISCUSIÓN}

\section{Inversión de ondas de cuerpo de Kikuchi y Kana- mori}

La inversión a menudo se convierte inestable; Debido al complejo equilibrio entre el mecanismo, la localización y la sincronización de los subeventos. La solución es no lineal y no única, por lo tanto, no es posible determinar "la mejor" solución de la inversión de forma de onda, debido a que diferentes secuencias pueden explicar igualmente bien los mismos datos. Bajo estas circunstancias es esencial primero investigar las características de todas las posibles soluciones.

El sismo de Armenia del 25 de Enero de 1999 en el departamento del Quindío, cuya longitud y trayectoria de ruptura fue obtenida mediante un análisis de ondas de cuerpo telesísmicas, el momento sísmico se obtuvo con la modelación del tren de ondas $\mathrm{P}$ y $\mathrm{SH}$, para un momento sísmico total de $1.26 \times 10^{25}$ dina* $\mathrm{cm}$ el cual es alrededor de $40 \%$ más pequeño que el momento sísmico de $2.1 \times 10^{25}$ dina* $\mathrm{cm}$ obtenido por Monsalve y Vargas (2) presentando una regular coincidencia; y alrededor de un $37.31 \%$ menor que el momento sísmico de $2.01 \times 10^{25}$ dina*cm reportado por Harvard-CMT. Dicha diferencia es debida probablemente a la estructura fuente y receptor utilizados en estos estudios.

La solución de Monsalve y Vargas (2002) indica un único subevento de rumbo con componente normal, mientras que la solución obtenida en este proyecto muestra dos subeventos siendo predominante el mecanismo de rumbo con componente normal pues $\lambda=-166.7^{\circ}$ y $\delta=$ $48.9^{\circ}$. Dicha discrepancia es debida principalmente en la forma en que la secuencia de ruptura es dividida en subeventos.

Para el proceso de inversión de forma de onda fue necesario restringir el plano de falla a anchos menores o iguales a $10 \mathrm{~km}$ y longitudes entre 10 y $20 \mathrm{~km}$, puesto que en el estudio de Monsalve y Vargas (2), las réplicas estaban distribuidas en un área de ruptura obtenida de $124 \mathrm{~km}^{2}$ (plano de ruptura de 10.6 x $11.7 \mathrm{~km}$ ) La relación entre ruptura y momento sísmico que se predice en el estudio de Somerville (20) establece:

$$
A=1.30 \times 10^{-18} * M_{0}^{0.79}
$$

Con el momento sísmico obtenido en la inversión de onda para el presente estudio, la ecuación [19] estimaría un área de ruptura de $88 \mathrm{~km}^{2}$, que en términos generales está relativamente de acuerdo con la distribución de réplicas en el área de ruptura del sismo. Para la solución final, el área total de ruptura obtenida por el proceso de inversión fue de 125 $\mathrm{km}^{2}$ (plano de ruptura de $12.5 \times 10 \mathrm{~km}$ ).

\section{Cálculo de sismogramas sintéticos de movi- miento del suelo}

De acuerdo a las modelaciones obtenidas se pudo determinar que existe una aceptable coincidencia entre la señal simulada y la señal registrada, siendo más congruente la señal simulada desde el observador Andes que la simulada desde Perero, probablemente debido a un efecto de sitio en la zona del observador (señal registrada), reflejados en la amplificación en la amplitud de la señal sísmica, debido a la influencia de las condiciones geológicas y topográficas. La respuesta y la magnitud de esa amplificación está controlada principalmente por las propiedades geotécnicas de los materiales geológicos, especialmente por sus características dinámicas, así como el espesor de las capas y la profundidad a la que se encuentra el sustrato rocoso $(23,24)$.

La no coherencia de las señales (simuladas y registradas) puede deberse además al modelo de velocidad 1D utilizado para obtener la señal simulada puesto que se suponen capas homogéneas del suelo e idealizan las condiciones del mismo.

\section{CONCLUSIONES}

- La inversión de forma de onda permitió establecer un área de ruptura de $125 \mathrm{~km}^{2}$ en un rectángulo de $10 \times 12.5 \mathrm{~km}$, que es similar a la obtenida por Monsalve y Vargas (2) de $124 \mathrm{~km}^{2}$ en un rectángulo de 10.6 x $11.7 \mathrm{~km}$, utilizando una función temporal triangular de base $9 \mathrm{~s}$, equivalente al tiempo de ruptura y tiempo de levante (rise time) de $4.5 \mathrm{~s}$. Esta área corresponde a la falla Córdoba (segmento Pijao-33), con una dislocación obtenida de $\Delta \mathrm{u}=$ $0.324 \mathrm{~m}$. 
- El mecanismo obtenido en el proceso de inversión corresponde a la sumatoria de dos subeventos, el cual corresponde a una falla normal con desplazamiento lateral izquierdo, con un azimut $\varphi=111.5^{\circ}$, un buzamiento $\delta=48.9^{\circ}$, un vector de deslizamiento con un ángulo $\lambda=-166.7^{\circ} \mathrm{y}$ un momento sísmico de $\mathrm{M}_{0}=1.26 \times 10^{25}$ dina* $\mathrm{cm}$, que corresponde a un $\mathrm{M}_{\mathrm{w}}=6.0$.

- La diferencia existente entre el momento sísmico ( obtenido por Monsalve y Vargas2) haciendo uso del método de inversión de ondas de cuerpo propuesto por Nabelek y el momento sísmico obtenido por el método de inversión de ondas de cuerpo telesísmicas propuesto por Kikuchi y Kanamori, es de alrededor del 40\% (siendo menor el obtenido por este ultimo) es debido probablemente al modelo de estructura de la fuente y receptor utilizado durante el análisis; además, el método propuesto por Kikuchi and Kanamori permite tener libertad en la variación y secuencia de los subeventos durante el proceso de inversión generando una componente anisotrópica o dipolo no lineal compensado (CLVD) que genera la no linealidad en el doble par que es tenida en cuenta en este método; además, la suma del momento sísmico de los subeventos encontrados no es hecha de manera lineal.

- La diferencia existente entre el momento sísmico obtenido por Harvard - CMT calculado usando ondas superficiales de periodo largo y el momento sísmico obtenido por el método de inversión de ondas de cuerpo telesísmicas propuesto por Kikuchi y Kanamori, es de alrededor del 37.31\% (siendo menor el obtenido por este ultimo) es debido probablemente al modelo de estructura de la fuente y receptor utilizado durante el análisis.

- El método propuesto por Kikuchi and Kanamori es aplicable a sismos con magnitud $\mathrm{M}_{\mathrm{w}}>6$, siendo consistente con la magnitud encontrada en este análisis de $\mathrm{M}_{\mathrm{w}}=6$.

- La "mejor" solución del proceso de inversión de ondas de cuerpo telesísmicas depende de las restricciones que sean impuestas en este proceso, para esto, se deben tener en cuenta los resultados obtenidos en otros estudios (haciendo uso de diferentes métodos de inversión) de análisis de ondas superficiales de periodo largo, geodesia y geológicos.
- De acuerdo a los resultados obtenidos en este análisis, es posible concluir que el método de inversión de ondas de cuerpo telesísmicas propuesto por Kikuchi and Kanamori es recomendable para futuros estudios, puesto que con este se obtiene el mecanismo y patrón de ruptura de eventos complejos. La ruptura es presentada por una secuencia se subeventos distribuidos en el plano de falla, los mecanismos se permiten que varíen durante la secuencia (aunque esto puede inestabilizar la inversión) pero reproduce fielmente el proceso de ruptura de la fuente sísmica; además, es posible utilizar ondas P, SH, SV y PP invertidas simultáneamente. En otros métodos como el de Nabelek (4) la inversión se realiza en el dominio del tiempo para determinar algunas de las complejidades de la función fuente, esta la mayoría de veces resulta extremadamente compleja; además, el mecanismo se determina por el método iterativo de mínimos cuadrados. En el método de Barker y Langston (5) se invierte el momento sísmico y se obtienen diferentes mecanismos que explican igualmente bien los mismos datos, por lo tanto, es necesario imponer una restricción en la solución.

- El mayor número de eventos se concentró hipocentralmente entre $10 \mathrm{~km}$ y $20 \mathrm{~km}$ y sus mecanismos focales de rumbo e inversos. Pudo distinguirse también, un grupo de sismos cuyos mecanismos focales normales, se ubicaron en profundidades menores a los $10 \mathrm{~km}$; sugiriendo un proceso distensivo superficial en la zona, donde son movilizados los segmentos de falla más superficiales.

- La simulación de señales obtenidas mediante proceso de inversión de forma de onda son comparables (amplitud y forma) con las registradas por IRIS, excepto la componente $\mathrm{SH}$ de las estaciones: SCZ, PFO y TSUM.

- Con la simulación de señales utilizando algoritmo de Spudich y Xu (14) se obtuvo una buena coincidencia en las amplitudes en velocidad de las señales simuladas y registradas. Por lo tanto, este puede ser utilizado para estimar las aceleraciones que podrían suceder en determinada zona referente a una falla y a una magnitud. Lo anterior, teniendo en cuenta características de la fuente previamente obtenidos en la inversión de forma de onda. 


\section{AGRADECIMIENTOS}

Agradecemos a la red Incoporated Research Institutions for Seismology (IRIS) por facilitar las trazas telesísmicas de las dieciséis estaciones utilizadas en este proyecto.

\section{BIBLIOGRAFIA}

1. Monsalve H y Vargas C, El sismo de Armenia (Colombia) del 25 de enero de 1999: Un análisis telesísmico de ondas de cuerpo, observaciones de campo y aspectos sismotectónicos. Revista Geofísica 57 2002; 21-57.

2. Gil F, Bohórquez O, Monsalve H, Osorio J.A, Ospina L.M, Acevedo A y Sanz L. Análisis de la secuencia de réplicas del sismo del 25 de Enero de 1999, con base en una red sismológica local (enero 26 - marzo 31, 1999), I Seminario Internacional de Riesgo Sísmico 1999; sep. 16-19.

3. Monsalve H, Geometría de la subducción de la Placa Nazca en el Noroeste de Colombia: Implicaciones tectónicas y sísmicas. Instituto de geofísica UNAM. Tesis de Maestría 1998; 107 pp.

4. Nabelek, J.L, Determination of earthquake source parameter from inversion of body waves, Phd. Thesis, MIT, Cambridge, Masachusetts 1984.

5. Barker, J and C.A. Langston, Moment tensor inversion of complex earthquakes. Geophys. J. R. Astr. Soc. 68 1982; 777-808.

6. Koyama J, Time-dependent moment tensor inversion for the 1983 Japan sea earthquake. Zisin 2, 40 1987; 405-416 (in Japanese with English abstract).

7. Hirata K y I Kawasaki, Space-time dependent moment tensor, Programme and Ab-stracts seism. Soc. Japan, $\mathrm{N}^{\circ} 2$ 1988; 16 (in Japanese).

8. Kikuchi M and Kanamori H, Inversion of complex body waves-III. Bull. Seismolog. Soc. Am.,81 1982; 2335-506.

9. Kikuchi M and Kanamori, H, Inversion of complex body waves. Bull. Seismolog. Soc. Am.,72 1991; 491-506.

10. Bouchon M, Teleseismic body wave radiation from seismic source in a layered médium, Geophys. J. R. Astr. Soc. 47 1976; 515-530.

11. Haskell N.A, Crustal reflection of plane SH waves, J. Geophys. Res., 65 1960; 4147-4150.

12. Haskell N.A, Crustal reflection of plane P and SH waves, J. Geophys. Res., 67 1962; 4751-4767.

13. Plafker G, Tectonic aspects of the Guatemala earthquake of 4 February 1976. Science 93 1976; 1201 1208.

14. Spudich P and Xu L, Documentation of software package Compsyn sxv3.11: programs for earthquake ground motion calculation using complete 1-d green's functions. International Handbook of Earthquake and Engineering Seismology, edited by W. H. K. Lee, Kanamori, H., Jennings, P., Kisslinger, c. Academic Press 2002.

15. SAC (Seismic Analysis Code) (v101.5c - Febrero 2012)

16. Pardo A, Análisis determinista de la amenaza sísmica para el Eje Cafetero. Tesis de grado. Universidad del Quindío. Programa de Ingeniería Civil 2011.

17. Jeffreys H. and K. E. Bullen, Seismological tables. Office of the British Association, Burlington House, London. 1958.

18. Aki K. and Richards P.G, Quantitative Seismology. Theory and Methods, W.H. Freeman and Company, San Francisco, 1980; 557 pp.

19. Das S and Aki K, Fault planes with barriers: A versatile earthquake model. J. Geophys. Res. 82 1977; 5648-5655.

20. Somerville P, K. Irikura, S. Sawada, D. Wald, N. Abrahamson, Y. Iawasaki, N. Smith, A. Kowada, Characterizing crustal earthquake slip modls for the prediction of strong ground motion. Sesismological Research Letters 70 1999; 1, 59-79.

21. París G, Mapa Neotectónico Preliminar del Quindío- rasgos morfoneotectónicos, Proyecto fallas potencialmente sismogénicas. Alcaldía de Armenia 1997 
22. Guzmán J, Franco G. y Ochoa M, Proyecto para la mitigación del riesgo sísmico de Pereira, Dosquebradas y Santa Rosa de Cabal - Evolución geotectónica, Carder 1997; 80 pp.

23. Aki K, Local site effects on strong ground motion. Reprinted from proceedings of earthquake engineering and soil dynamics II, GT Div / ASCE, Utah 1988; pp. 103-155.

24. Borcherdt R.D, Estimates of site-depent response spectra for desing (methodology and justification). Earthquake Spectra. 10 1994; pp. 617-673. 